\title{
El empleo de las TICS en la gestión pública
}

\author{
Raúl Villalobos Jaquehua \\ Raulv14@hotmail.com \\ Universidad César Vallejo \\ Lima - Perú \\ Fernando Emilio Escudero Vílchez \\ fescudero@silfersystem.com \\ Universidad César Vallejo \\ Lima - Perú \\ Silvia Liliana Salazar Llerena \\ ssalazar@silfersystem.com \\ Universidad César Vallejo \\ Lima - Perú
}

\section{RESUMEN}

OBJETIVO: La actual investigación sostuvo como propósito inicial el de revisar las estrategias que se optaron para poder visualizar el empleo de las TIC dentro de la gestión pública, debido a todos los trabajos ineficientes que se hace ahí y dentro de todos los sectores que este abarca. Una estrategia es un plan de acción que se puede realizar, indistintamente sea por una persona o un grupo de personas, de largo o corto plazo para que de dicha forma se pueda cumplir todas las metas que se tienen previstas, así como los objetivos. METODOLOGÍA: La metodología empleada dentro del presente artículo ha sido bajo un enfoque cuantitativo, mediante la revisión de variada bibliografía de organismos nacionales e internacionales como también autores referentes a la información previamente investigada por ellos. RESULTADOS: Como resultado se evidenció que el empleo de las TICS en la gestión pública permite llevar a cabo planes, estrategias y políticas nacionales que estén dirigidas a satisfacer las necesidades que presenta la ciudadanía. CONCLUSIÓN: Finalmente, se concluye que, el empleo de las TIC es vital para nuestra gestión pública, ya que en nuestro país cuenta con enorme retraso a comparación de otros países que afecta a todos los sectores y especialmente a la gestión pública y, por último, que el estado intentará resolver este problema que viene afectando desde años atrás.

Palabras clave: gestión pública; tecnologías de información; ciudadanía 


\title{
The use of TICS in public management
}

\begin{abstract}
OBJECTIVE: The main purpose of this research was to review the strategies that were chosen to be able to visualize the use of ICT within public management, due to all the inefficient work that is done there and within all the sectors that this covers. A strategy is an action plan that can be carried out, either by a person or a group of people, in the long or short term so that in this way all the goals that are planned can be met, as well as the objectives. METHODOLOGY: The methodology used in this article has been under a quantitative approach, through the review of various bibliography of national and international organizations as well as authors referring to the information previously investigated by them. RESULTS: As a result, it was evidenced that the use of ICTs in public management allows carrying out national plans, strategies and policies that are aimed at satisfying the needs of citizens. CONCLUSION: Finally, it is concluded that the use of information and communication technologies (TIC) is vital for our public management, since in our country it has an enormous delay compared to other countries that affects all sectors and especially public management and, finally, that the state will try to solve this problem that has been affecting for years.
\end{abstract}

Keywords: public management; information technologies; citizenship

Artículo recibido: 02 noviembre. 2021 Aceptado para publicación: 28 noviembre 2021

Correspondencia: Raulv14@hotmail.com Conflictos de Interés: Ninguna que declarar 


\section{INTRODUCCIÓN}

La gestión pública es el órgano que administra una empresa o estado de gran relevancia, buscando brindar y mejorar sus servicios a las personas. Es por ello que dentro de la gestión pública se observa las variedades de estrategias y herramientas que implementa para mejorar la efectividad y eficacia de los servicios que ofrecen, mejorando la gestión y proceso de trámites, el empleo de tecnologías con el objetivo de realizar las áreas de trabajo con mayor efectividad, y además mejorar las capacidades de los colaboradores mediante las tecnologías, mejorando la calidad que se brinda por lo que es importante recalcar si la gestión pública tuviera una mala organización y/o administración, ya sea para cualquiera de sus distintos sectores, la efectividad y eficiencia de estos, sería pésima y se tendría un retraso con los objetivos que se la población esperaba (Machín et al., 2019).

Para Livari et al. (2020) indica que la tecnología en la sociedad debe ser incentivada por los poderes gubernamentales, siendo ellos que promuevan mediante la gestión pública integrar y socializar dentro de los ciudadanos, permitiéndole conocer el desarrollo del uso de las tecnologías en la sociedad; teniendo como fin poder mejorar la calidad de vida, la cual estará destinada en ofrecer mayor información directa a los ciudadanos.

La gestión pública nace como una alternativa para los gobiernos que ambicionan reforzar a la democracia representativa, debido a que en esta se refleja que existe una gran carencia en el marco de la aceptación por parte de los ciudadanos; es decir, se desconfía de los gobiernos. Del mismo modo menciona que la gestión pública en América Latina es importante aplicarla debido a sus grandes beneficios que se define como una agenda que involucra cambios en diferentes ámbitos de gestión pública, los que abarca los diversos sistemas de planeación y los mecanismos de gestión (Pliscoff, 2017). En la misma linea,los autores Hodges y Howieson et al. (2017) mencionan que debido a los cambios que han estado experimentado los cambios sociales, políticos y económicos se debe implementar de manera optima las herramientas tecnologicas a fin de poder conseguir la participaciòn de la ciudadania.

Además, aporta Marulanda et al. (2017) que en la actualidad con el desarrollo inmenso que tiene la tecnología abarcando diversas áreas de trabajo, esta puede beneficiar en la productividad, efectividad que tendría el trabajo; especificando que el objetivo principal no es mejorar ampliamente las herramientas digitales, si no por lo contrario mejorar las 
capacidades de los trabajadores con las tecnologías obteniendo mayor efectividad de la organización en la gestión pública. Y justamente, lo que dice el investigador es altamente acertado, ya que uno no busca específicamente hacer una enorme mejora en las TIC, sino busca hacer una mejora en las propias labores que cada individuo tiene y de dicha forma, poder mejorar como sociedad.

Los autores Asmat et al. (2019) afirman que, aunque se haya incrementado la producción científica sobre las TIC durante estos últimos años, la producción que ha estado haciendo el Perú es indudablemente bajo si es que lo comparamos con otros países más desarrollados y subdesarrollados. El hecho de que algunas instituciones nacionales hayan participado es sólo un referente, ya que se les es posible hacernos la muestra de que hay una amplia cantidad de diferencias entre hospitales, universidades, consultoras que desde hace ya en los últimos 10 años vienen haciendo la implementación de investigar en TIC. En efecto, dicho autor menciona una verdad triste para nuestro país, y es que, es inevitable el no comparar el avance que se tiene en el Perú con los países primermundistas o como ya se había mencionado, con países aledaños como es el caso de Chile, que nos supera aproximadamente 50 años en el desarrollo como nación.

La revisión sistemática de Espinoza et al. (2019) evaluó que es posible encontrar la existencia de estudios junto con evaluaciones que pudieron hacer posible la muestra del potencial que tienen las intervenciones con el empleo de las TIC, propiamente el uso de dispositivos móviles, todo esto con el único fin de poder hacer una mejora en la salud infantil y la salud materna dentro de países subdesarrollados. Un claro ejemplo es el uso de mensajes de texto, ya que existe evidencia de que dichos mensajes pueden mejorar la unión a un tratamiento alguno y también, poder realizar la organización y asistencia médica en distintas condiciones que se tenga de salud por medio de recordatorios. Se añade que, haciendo uso de dichos métodos se pudo ver un claro aumento en la adherencia a los tratamientos (40\%), mientras que en las asistencias a citas médicas aumentó un 18\% y se hizo una disminución de las cancelaciones en $18 \%$. Como otros puntos positivos, se tiene el hecho de que se redujo el porcentaje de dosis no tomadas, todo esto ayuda a que se reduzcan toda interrupción hacia los tratamientos. Tal y como se ha estado mencionando en todo este trabajo, el uso de las TIC es más que una ayuda, es altamente considerada como un apoyo en la gestión pública y dentro de los distintos sectores que 
esta tenga, como se pudo observar, el sector salud en área más necesitadas es un claro ejemplo de lo que se ha estado diciendo.

\section{ESTRATEGIAS METODOLÓGICAS O MATERIALES Y MÉTODOS}

El actual analisis se realizó con un enfoque cuantitativo,de acuerdo con Guedes dos Santos et al. (2017), señalo que una investigación cuantitativa se basa en la elaboración de estudios que están vinculados a datos númericos, los cuales fomentan la conjunción de detalles e información de esta manera permita corroborar teorias y/o hipótesis

En la misma linea Tobi y Kampen (2018) señalan que esta permitió evidenciar los resultados de manera numérica y cuantificar los productos en aspectos estadísticos, asimismo recopilar información a manera de verificar o corroborar las hipótesis y/o teorías planteadas.

\section{Técnicas e instrumentos de recolección de datos}

Por otro lado, los datos utilizados se han originado a partir de una relación hipotéticadeductiva, la que nos permitirá verificar la credibilidad de los mismos, siendo que la verificación será de manera empírica. Por ende, se refiere a las variables para poder alcanzar a una conclusión en este caso sería la hipótesis para verificar su credibilidad y exactitud, y en caso el resultado fuera optimo se concluiría que hay una relación con la teoría inicial y los planteamientos del problema de la investigación (Sánchez,2019).

Así también, los datos utilizados se han originado a partir de una relación hipotéticadeductiva, siendo así que la verificación será de manera empírica y las variables han sido estudiadas por la relación entre ellas, esto con la finalidad de obtener un mayor entendimiento y comprensión de las mismas en conjunto, así como de su importancia de investigación (Núñez,2017).

\section{Procedimiento}

Se realizó la búsqueda bibliográfica y sistemática de artículos científicos en las siguientes bases de datos: Redalyc, Scielo y Scopus. La búsqueda y selección de los artículos inició en el período 2017 hasta el año 2021, para la investigación se decidió la selección de los artículos bajo una estructura relacionada con las variables, que consistió en realizar una búsqueda de información enfocada en el uso de las TICS y la relación con la gestión pública en el Perú, sus características, normativa nacional, beneficios, enfocándonos en la incidencia de una variable sobre la otra. 
Es por ello que, los datos obtenidos tienen el objetivo de ser desarrollados de manera correcta en el presente trabajo, dado que son parte de una revisión bibliográfica ardua relacionada a la temática mencionada anteriormente, los criterios para su selección fueron la fecha de publicación, el contenido veraz y verificable de la información, así es como se desarrollan; esta será utilizada como sustento estadístico en el análisis que se llevará a cabo.

\section{RESULTADOS Y DISCUSIÓN}

\section{Resultados}

En base a toda la recolección de datos y búsqueda de investigaciones que fueron referentes en el tema, se pudo evidenciar que con el empleo de las Tics se relaciona de manera significativa con la gestión pública ya que permite llevar a cabo planes, estrategias y políticas nacionales que estén dirigidas a satisfacer las necesidades que presenta la ciudadanía, para lograr ello se debe contar con herramientas tecnológicas de información que permitan informar a la ciudadanía, puesto que se le pueda orientar de una manera más sencilla sobre la acción política.

Es importante señalar que la transformación digital en la gestión pública es realmente importante, dado que actualmente debido a la pandemia, todo se realiza de manera virtual haciendo empleo de las herramientas informáticas para desarrollar sus actividades fundamentales como el poder trabajar, estudiar y comunicarse ,por lo cual, constituye un problema o barrera para los ciudadanos el que no todos puedan tener acceso a los medios digitales, para lo cual se deberían plantear estrategias por parte del gobierno, en favor de los ciudadanos y su desarrollo en la sociedad.

Por otro lado la TIC se relaciona con los sistemas de apoyo a la planificación generalmente han promovido gobiernos y servicios inteligentes, participación electrónica y una colaboración participativa. Esto podría conducir a cambios graduales en las organizaciones gubernamentales, nuevos lazos con gobiernos, el sector privado y la ciudadanía, y mejoras en la ciudad.

Finalmente, el empleo de las TIC al apoyo de la dirección pública se encarga de abastecer servicios públicos de manera más eficiente puesto que está dirigido al ciudadano. Por eso contar con este instrumento mejora y reduce el curso de soporte institucional, que proporcionan la creación de canales que posibiliten el aumento de la transparencia y la participación ciudadana. 


\section{Discusión}

En función del objetivo planteado en esta investigación, resaltar la implementación de las herramientas tecnológicas en relación a la gestión pública actual, se expondrán los resultados de las investigaciones de 9 artículos, los cuales poseen características e información importante para su desarrollo, pero sobre todo que contribuyen a la realización de este artículo de revisión.

Mukhtarov et al. (2018) entre sus resultados precisaron que la participación pública es un tema importante en la gestión pública con la difusión de las TIC, la gestión pública ha experimentado cambios importantes, incluido el proceso y los resultados de la participación pública. Por otro lado, se menciona que las herramientas TIC han permitido que los ciudadanos estén informados, además, tienen el potencial de contribuir a la eficiencia y eficacia de la prestación de servicios básicos.

Cohen et al. (2017) refieren que el uso de las TICS e internet impulsan el interés de los ciudadanos en la determinación de decisiones por parte de los mandatarios, lo que a su vez los motivaría potencialmente a tener una intervención más activa en los asuntos públicos. Por ende, se pudo evidenciar que hay una relación colaborativa entre las TIC que es un medio para informar a la ciudadanía de una manera más sencilla y colaborativa para incentivar su participación democrática.

McBride \& Draheim (2020) afirman que debe existir una relación entre la gestión pública y los medios tecnológicos con el fin de integrar una dinámica que favorezca a la ciudadanía y cumpla las expectativos que estos presentan sobre la gestión que se realiza. Por otro lado, enfatizan en que la implementación de las TICS implementa en la gestión pública, debido a que las operaciones y funciones probablemente puedan ejecutarse de manera sistemática y agregar nuevas opciones positivas a los funcionarios públicos, con el uso de las TICS.

Díaz (2017) señala que cada estado es responsable de sus ciudadanos por lo tanto estos mismos deben de aprovechar que la evolución y los avances de las tecnologías le brindan a uno una ventaja favorable en la utilización de estas herramientas. Esta es una herramienta que contribuye directamente a un desarrollo mayor, pero es necesario comprender la prioridad de cada proposición. Es conveniente también que se considere los objetivos comunes que se desean cumplir y que estos representen un beneficio para toda la población, de esta manera se podrán evitar las desigualdades causadas por la 
modernización de ciertos sectores, integrando estos sectores olvidados por años para que estos puedan sentir los beneficios que se buscan aplicar.

Jiménez et al. (2017) precisan que para la implementación de un gobierno electrónico es fundamental tomar en cuenta las TIC'S, las cuales necesitan estar en óptimas condiciones para desempeñar su función de manera efectiva, esto teniendo en cuenta que el gobierno electrónico tiene como finalidad maximizar el alcance a la ciudadanía de los servicios públicos, así como mejorar la gestión nacional y con ello lograr un país socio-productivo. Es por esto que su implementación en distintos ámbitos nacionales, como la educación y la salud, es tan importante. Además, la capacitación a los funcionarios que harán uso de estas herramientas también debe ser prioridad del Estado, esto con la finalidad de aprovechar los recursos con los que se cuenta.

Valenzuela et al. (2021) señalan que, a comparación de años anteriores, en el actual el brindado de información y la estable comunicación en el sector público, es por ello que analiza el impacto que podría tener la implementación de tecnologías; en lo cual ayudaría en las áreas de la gestión pública, administrando y comunicándose de manera directa hacia los ciudadanos y que estos puedan realizarlo mediante dispositivos o herramientas electrónicas impuestas por el estado. Además de permitir la efectividad y eficacia en la labor dentro de las instituciones públicas e incentivando a que los ciudadanos puedan formar parte y de tomar decisiones del recurso público. De dicha forma, también es necesario añadir que nuestro país, a comparación de los países más desarrollados o incluso de la misma Latinoamérica, está bastante retrasado con el uso de las TIC, especialmente en lo que es sector salud o el sector educativo.

Moreira y Lima (2019) señalan en una de sus conclusiones las TICS se exhibe como un principio fundamental para que la gestión pública logre sus objetivos. Asimismo, la transparencia y el control, adecuados se presentan como factores fundamentales para poder potenciar los frutos en el sector público, ya que conducen a los gestores públicos a buenas prácticas gerenciales. Los resultados indican que las TICS y la efectividad de la gestión pública predomina positivamente en el progreso socioeconómico municipal, lo que interpreta como las facilidades que se le deben dar a la ciudadanía para informarse mediante las herramientas tecnológicas.

Anaya et al. (2021) mencionan que la gestión pública para ser eficaz y eficiente para brindar respuesta de solución a la ciudadanía se tiene que implementar los recursos como 
son las herramientas de información y comunicación para perfeccionar la provisión de servicios públicos, la gestión de los procesos y el fortalecimiento de capacidades de los funcionarios y de la ciudadanía con el fin de que pueda cumplir con las obligaciones concedidas.

Veale y Brass (2019) refieren que buscan utilizar nuevas formas o implementar nuevas herramientas para proporcionar mejores servicios públicos, las reformas más significativas han sido las TICS para que se pueda mejorar la eficiencia del gobierno mejorando la gestión y prestación de servicios públicos para el desarrollo continuo del país beneficiando a todos los ciudadanos.

\section{CONCLUSIÓN O CONSIDERACIONES FINALES}

- Como primera conclusión, se ha podido llegar a que el uso de las TIC, en la gestión pública y en sus distintos sectores, influyen de una manera tan positiva que definitivamente tiene que estar incluida y actualizada de tal manera que permita la total eficiencia para dicha gestión y para todos los sectores que este abarque.

- Como segunda conclusión, se pudo llegar que la ausencia de dichas herramientas tecnológicas ha hecho un verdadero mal al país, contando todos los retrasos que este tiene frente a otros países de distintos continentes o incluso de países de la propia Latinoamérica; sin embargo, en un punto de nuestra historia actual se pudo tener un ascenso y mejora en los sectores públicos.

- Como tercera y última conclusión, se pudo llegar a que el virus del covid-19, obviamente, fue un retraso hacia nuestro país, por lo que, de dicha forma también, se tuvo un retraso enorme con la adquisición de nuevas TICS y, por ende, dentro de la gestión pública y los sectores que este tiene; sin embargo, el estado tomará cartas en el asunto para poder resolver o al menos intentar resolver dicho problema de la falta de las TIC, ya que se considera de alta emergencia.

\section{LISTA DE REFERENCIAS}

Anaya Figueroa, T., Montalvo Castro, J., Calderon, A., \& Arisque Albureque, C. (2021). Escuelas Rurales en el Perù:Factores que acentuan las brechas digitales en tiempos de pandemia y recomendaciones para para reducirlas. Educación, 30(58). doi::http://dx.doi.org/10.18800/educacion.202101.001

Asmat Vega, N., Borja Villanueva, C., Berny Torres, L., Lizarzaburu Aguinaga, D., \& Morillo Flores, J. (2019). Estudio bibliométrico de la producción científica sobre 
TIC en Perú (2010-2017). Propósitos y Representaciones, 7(2), 196-209. http://www.scielo.org.pe/pdf/pyr/v7n2/a08v7n2.pdf

Cohen , S., Mamakou, X., \& Karatzimas, S. (2017). IT-enhanced popular reports: Analyzing citizen preferences. Government Information Quarterly, 34(2), 283295. doi:https://doi.org/10.1016/j.giq.2017.04.003

Dìaz Aldret, A. (2017). Participación ciudadana en la gestión y en las políticas públicas . Gestión y Política Pública, 26(2), 341-379. doi:http://www.scielo.org.mx/pdf/gpp/v26n2/1405-1079-gpp-26-02-00341.pdf

Espinoza Portilla, E., Henriquez Suarez , M., \& Villanueva Cadenas, G. (2019). Oportunidades de aplicación de las tecnologías de la información y comunicación (TICs) para fortalecer la lucha contra la anemia en Perú. Acta Médica Peruana, 36(2), 125-156. http://www.scielo.org.pe/pdf/amp/v36n2/a12v36n2.pdf

Guedes dos Santos, J., Lorenzini Erdmann, A., Schilindwein Meirelles, B., Marcelino de Melo , G., Pecini da Cunha, V., \& Ratchneewan, R. (2017). Integrating Quantitative and Qualitative data in mixed methods research. Texto \& Contexto Enfermagem, 26(3). doi:https://doi.org/10.1590/0104-07072017001590016

Hodges, J., \& Howieson, B. (2017). The challenges of leadership in the third sector. European Management Journal, 35(1). doi:https://doi.org/10.1016/j.emj.2016.12.006

Jiménez Pitre, I., Martelo, R., \& Jaimes, R. (2017). Escuela de Gobierno basada en TIC: Determinada para la Accesinilidad e Integralidad del Empoderamiento Digital. $\begin{array}{lll}\text { Información } & \text { Tecnológica, } & \text { 75-86. }\end{array}$ https://scielo.conicyt.cl/pdf/infotec/v28n5/art10.pdf

Livari , N., Sharma, S., \& Leena Venta, O. (2020). Iivari , NDigital transformation of everyday life - How COVID-19 pandemic transformed the basic education of the young generation and why information management research should care? International Journal of Information Management, 55. doi:https://doi.org/10.1016/j.ijinfomgt.2020.102183

Machín Hernández, M., Sánchez Vignau, B., López Rodríguez, M., \& Puentes Alvarez, P. (2019). La gestión pública local como garante de la eficacia en la administración pública cubana. Cooperativismo y Desarrollo, $7(2)$. 
http://scielo.sld.cu/scielo.php?script=sci_arttext\&pid=S2310$340 \times 2019000200212$

Maralunda Echeverry, C., López Trujillo , M., \& Valencia Duque, F. (2017). Gobierno y gestión de TI en las entidades públicas. Ad-Minister(31), 75-92. http://www.scielo.org.co/pdf/adter/n31/1692-0279-adter-31-00075.pdf

McBride, K., \& Draheim, D. (2020). On Complex Adaptive Systems and Electronic Government: A Proposed Theoretical Approach for Electronic Government Studies. The Electronic Journal of e-Government, 18(1), 43-53. doi:10.34190/EJEG.18.1.004

Moreira da Silva, C., \& Lima Crisóstomo, V. (2019). Fiscal management, public management efficiency and socioeconomic development of Ceará municipalities. Brazilian Journal of Public Administration, 53(4). doi:https://doi.org/10.1590/0034-761220180234

Mukhtarov, F., Dieperink, C., \& Driessen, P. (2018). The influence of information and communication technologies on public participation in urban water governance: A review of place-based research. Environmental Science \& Policy, 89, 430-438. doi:https://doi.org/10.1016/j.envsci.2018.08.015

Núñez Moscoso, J. (2017). Mixed methods in education research: towards a reflexive use. Cadernos de Pesquisa(47). doi:https://doi.org/10.1590/198053143763

Pliscoff Varas, C. (2017). Implementing the new public management:problems and challenges to public ethics. Convergencia, 24(73). http://www.scielo.org.mx/scielo.php?pid=S1405$14352017000100141 \&$ script=sci_abstract\&tlng=pt

Sànchez Flores, F. (2019). Fundamentos epistémicos de la investigación cualitativa y cuantitativa: Consensos y disensos. Revista Digital de Investigación en Docencia Universitaria, 13(1), 102-122. doi:http://dx.doi.org/10.19083/ridu.2019.644

Tobi , H., \& Kampen, J. (2018). Research design: the methodology for interdisciplinary research framework. Quality \& Quantity, 52, 1209-1255. doi:https://doi.org/10.1007/s11135-017-0513-8

Valenzuela Fernández, L., Collantes Inga, Z., \& Durand Hipólito, E. (2021). Sobre la gobernanza digital, política digital y educación. Revista eleuthera, 22(2). 
http://www.scielo.org.co/scielo.php?script=sci_arttext\&pid=S201145322020000200088

Veale, M., \& Brass, I. (2019). Administration by Algorithm? Public Management Meets Public Sector Machine Learning. Algorithmic Regulation. https://papers.ssrn.com/sol3/papers.cfm?abstract_id=3375391 